\title{
Diversidad de insectos acuáticos como bioindicadores de la calidad del agua de la microcuenca del río jutiapa en las quebradas corralitos, limones y jutiapa del parque nacional La Tigra, Francisco Morazán, Honduras
}

Alba Isbela Hernández O. ${ }^{*}$, Rosella Martínez, Delia Moreno, Luis Martínez

\section{RESUMEN}

El interés por conocer el estado actual de los cuerpos acuáticos y su evolución en el tiempo, ha estimulado una fuerte investigación durante las dos últimas décadas en la búsqueda de establecer estándares de juicio de "calidad de agua" que permitan satisfacer las demandas de uso del recurso. Una aproximación puede ser abordada a través del análisis de las características físico-químicas del agua y por otro lado, a través del uso de organismos indicadores de la calidad ambiental. En el período comprendido entre febrero y marzo (época seca) del 2009 se realizó una colecta de insectos acuáticos en la quebrada Limones, quebrada Corralitos y el río Jutiapa en el Parque Nacional La Tigra, Francisco Morazán, Honduras.

El objetivo del estudio fue determinar la abundancia y la diversidad de insectos acuáticos bentónicos como bioindicadores de la calidad del agua y establecer las posibles relaciones con los parámetros físico-químicos de los sitios antes mencionados, mediante el análisis de parámetros comunitarios (Índice de Diversidad Shannon-Wienner, Índice de Berger-Parker, Índice de Similitud de Jaccard de la macrofauna bentónica y la aplicación del Índice Biótico de Familias BMWP-CR) para determinar la calidad del agua de las quebradas seleccionadas. En la quebrada Corralitos se colectaron 371 ejemplares, en la quebrada Limones 177 y en la quebrada Jutiapa 64, con un total de 612 especímenes, de 8 órdenes y 26 familias. El orden Díptera con 6 familias (siendo las principales Culícidae con $31 \%$ y las familias Simúlidae y Leptophlebiidae con $18.79 \%$ ) representó el $51.96 \%$. Los órdenes con menos especímenes fueron Lepidóptera (0.495\%) y Plecóptera $(0.16 \%)$.

La quebrada más diversa fue la quebrada Limones y la menos diversa fue la quebrada Jutiapa. El mayor índice de dominancia fue para la quebrada Jutiapa, siendo la familia Leptophlebiidae más dominante. El índice de Similitud resultó ser

Universidad Nacional Autónoma de Honduras. Facultad de Ciencias. Escuela de Biología. albaoviedo2001@yahoo.com; rosellamartinez@hotmail.com; moreca@hotmail.com; luibiologia@gmail.om 
mayor entre la quebrada Limones y la quebrada Jutiapa. El uso del Índice Biótico de familias permitió definir a las 3 quebradas de la microcuenca Jutiapa como de aguas de calidad regular, eutrofia y contaminación moderada con muy poca diferencia en los valores calculados, pero siempre dentro del mismo rango. De las 3 quebradas, la que presentó mayor contaminación orgánica fue la quebrada Corralitos, ubicada en la parte media, donde es impactada por diferentes cultivos, especialmente de café. Los resultados del índice de diversidad indican que la quebrada Limones podría ser considerada como un sitio de referencia por las buenas condiciones en su calidad biológica para futuras evaluaciones.

Una comparación con los parámetros físicos y químicos del agua, permiten validar el uso del índice Biótico de Familias como una herramienta útil para la evaluación de la calidad de las aguas en ecosistemas de aguas continentales, por lo que se recomienda realizar estudios de la abundancia y de la diversidad de insectos acuáticos durante las diferentes épocas estacionales.

Palabras clave: Calidad de aguas continentales, bioindicadores de la calidad del agua, macroinvertebrados bentónicos, insectos acuáticos, Parámetros comunitarios, Índice de Diversidad Shannon-Wiener (H'), Índice de BergerParker, Índice de Similitud de Jaccard de la macrofauna bentónica, índice Biótico de Familias, BMWP, Parque Nacional La Tigra.

\section{ABSTRACT}

The interest in knowing the current status of inland water bodies and their evolution over time has stimulated research over the past two decades, seeking to establish standards of "water quality" that would meet the demands of resource use. In that sense, an approximation can be addressed through the analysis of physic-chemical characteristics of water and the use of indicator organisms in environmental quality. In the period between february and march (dry season) of 2009 was made a collection of aquatic insects in the Limones stream, Corralitos stream and the Jutiapa river in the La Tigra National Park, Francisco Morazan, Honduras.

The study's objective was to determine the abundance and diversity of benthic aquatic insects as bioindicators of water quality and to establish possible relationships with physicochemical parameters of the above sites, by analyzing community parameters (Shannon-Wiener Diversity Index, Berger-Parker index, Jaccard similarity index of the benthic macrofauna and implementation of the Family Biotic Hint BMWP-CR) to determine the water quality of selected streams. In Corralitos stream 371 specimens were collected, in Limones stream 177 and in 
Jutiapa river 64 , with a total of 612 specimens, 8 orders and 26 families. The order Diptera with 6 families (being the main Culicidae with 31\%, and the families Leptophlebiidae Simulidae with $18.79 \%$ ) accounted for $51.96 \%$. The orders with less especimens were Lepidoptera $(0.495 \%)$ and Plecoptera $(0.16 \%)$.

The water body with more diversity was Limones stream and the less diverse was the Jutiapa river. The dominance index was higher for the Jutiapa stream, being the most dominant Leptophlebiidae family. The similarity index was greater between the $q$ Limones stream and Jutiapa river. Use of the Biotic Index of families helped to define the 3 streams of the watershed Jutiapa with water quality as regular, moderate eutrophication and pollution with little difference in the calculated values, but within the same range. Of the 3 streams, which had higher organic pollution was the Corralitos stream, located in the middle, where it is impacted by different crops, especially coffee. The diversity index results indicate that the Limones stream could be considered as a reference site for good quality biological conditions for future assessments.

A comparison with the physical and chemical parameters of water, allows the validation of the use of the Family Biotic Index as a useful tool for the assessment of water quality in inland water ecosystems, so it is recommended to study the abundance and diversity of aquatic insects during different seasonal periods.

Key words: Inland water quality, bio-indicators of water quality, Inland water benthic macroinvertebrates, aquatic insects, Community parameters, Shannon-Wienner Diversity index, Berger-Parker index, Jaccard similarity index of the benthic macrofauna, Biotic Families Index, BMWP, La Tigra National Park. 


\section{INTRODUCCIÓN}

Una cuenca hidrográfica es un sistema natural dinámico de elementos físicos, biológicos y humanos que reaccionan equilibradamente entre sí, creando por lo tanto un conjunto único e indisociable en permanente evolución. La cuenca también es un medio humano de transformación, ya que representa el área en donde se ubican las actividades del hombre.

La conservación de los ecosistemas es un objetivo ampliamente aceptado en el mundo, al menos teóricamente, y así ha sido recogido por la Unión Internacional para la Conservación de la Naturaleza y los Recursos Naturales, a través de las principales estratégicas propuestas: mantener los procesos ecológicos y esenciales y los sistemas biológicos, preservar la diversidad genética y asegurar un aprovechamiento sostenido de las especies y de los ecosistemas. En el caso de los sistemas fluviales, su gestión y conservación para el aprovechamiento sostenido de los recursos que exigen adoptar una estrategia a nivel de cuenca hidrográfica, realizando una planificación del uso del suelo acorde con la conservación de los mismos, y teniendo en cuenta las interrelaciones que existen entre los componente fluviales y los sistemas terrestres que los rodean.

Gonzales del Tánago \& D.G de Jalón (1995), menciona que "un sistema fluvial ofrece al hombre no solo agua susceptible de ser embalsada y aprovechada para consumo doméstico y en la mayoría de actividades humanas, sino que el río ofrece también toda una serie de recursos y valores cada vez más apreciados, cuyo disfrute y conservación plantean un conflicto de usos del agua, que es necesario considerar en la planificación hidrológica de cada cuenca y resolver según objetivos, criterios de valoración y condiciones de partida distintos en cada río y, con mayor motivo muy diferentes de unas cuencas a otras".

Los ecosistemas acuáticos revisten un especial interés para el ser humano ya que el agua constituye un recurso imprescindible para el desarrollo de las actividades humanas. A su vez, los ecosistemas acuáticos constituyen en definitiva los colectores de las actividades agrícolas e industriales y desagües de las ciudades. (Roldán, 1999). En este caso, estas actividades contaminan las aguas que fluyen bajo las zonas de descarga a tal grado que limita el uso para actividades humanas a no ser que se sometan a tratamiento especial.

A nivel mundial es evidente el incremento en el deterioro de la calidad del agua de los ríos. En los países en desarrollo el problema se agrava, ya que las fuentes de financiamiento son insuficientes, así como las opciones o los recursos tecnológicos para el monitoreo, generalmente son irregulares en tiempo y calidad, por lo que la 
información generada carece de confiabilidad al pretender generar estrategias para el adecuado manejo de los recursos acuáticos y para el control de la contaminación.

De esta manera, ha resurgido el interés sobre técnicas de evaluación rápida para el monitoreo de la calidad del agua en varios países en desarrollo (Chessman, 1995). En esos métodos emplean a las comunidades biológicas existentes en el sistema acuático y hacen énfasis en el bajo costo de la investigación, un muestreo reducido y un análisis más eficiente de los datos.

Entre las comunidades más utilizadas están los macroinvertebrados, y su utilidad como indicadores para evaluar la calidad del agua se ha incrementado significativamente; su muestreo es simple y resulta menos costoso que la mayor parte de las técnicas analíticas de laboratorio (Payne, 1986; Chessman, B.C. 1995; Hilsenhoff, 1998). Además se encuentran en todos los sistemas acuáticos, por lo que favorecen los estudios comparativos, son de naturaleza sedentaria, lo que permite un efectivo análisis espacial de los efectos de las perturbaciones, presentan ventajas técnicas asociadas a los muestreos cuantitativos y análisis de las muestras, los que pueden ser realizados con equipo simple y barato, la taxonomía de muchos grupos está bien estudiada y existen numerosos métodos para el análisis de datos, incluyendo índices bióticos y de diversidad, así como de similaridad, etc., los cuales han sido utilizados ampliamente en biomonitoreos a nivel comunitario (Hellawell, 1986) y de respuestas individuales (Hawkes, 1979; Suess, 1982; Rosenberg et al., 1986).

En muchos países se ha adoptado su identificación, a nivel de familia, en estudios de monitoreo biológico para la evaluación de los grados de contaminación en ríos. Sin ser necesaria la identificación a nivel de especie (Payne, 1986; Chessman, B.C. 1995; Hilsenhoff, 1998).

Los conceptos biológicos de Forbes (1887) sobre comunidad, sentaron las bases para desarrollar las primeras ideas acerca de los biomonitoreos. En ese sentido las comunidades de plantas y animales podrían utilizarse para determinar el grado de contaminación orgánica en ríos. Este concepto posteriormente fue profundizado por Forbes y Richardson (1913). Forbes (1928) y Richardson (1928) clasificaron las zonas de contaminación a lo largo del Río Illinois en USA. Sin embargo, el primer uso de un índice biótico proviene de Alemania, a principios del siglo XX, cuando Kolkwitz \& Marsson (1909) desarrollaron la idea de la saprobidad (grado de polución) en ríos, como medida del grado de contaminación de aguas residuales, que provoca disminuciones del oxigeno disuelto y del efecto que esta disminución tiene en la biota de ríos (Cairns \& Pratt, 1993). 
Actualmente está bien establecido que la diversidad de plantas y animales, así como el número de individuos, dentro y en los alrededores de los cursos de agua son excelentes indicadores de su salud. Cuanto más diversas son las poblaciones mejor resulta la salud de estos ecosistemas. Si solo unas pocas especies están presentes, es muy probable que la corriente no sea saludable y esto se debe en gran parte a la cantidad de oxígeno disuelto, ya que este es un importante factor limitante en ecosistemas acuáticos.

En los ríos, la disolución del oxígeno se favorece de forma natural debido a la velocidad de la corriente. Sin embargo, cuando los niveles de materia orgánica aumentan debido a la descarga de desperdicios, ocurre una natural disminución del oxígeno disuelto que afecta, de manera variable, la fisiología de los individuos que componen la comunidad, es decir, la estructura de la comunidad se altera. Entonces, si empleamos métodos para medir cambios estructurales, ya sea la variación de la riqueza de especies y/o el número de individuos/especies, podremos tener una idea aproximada del estado en que se encuentra el ecosistema. Estas medidas son conocidas como índices biológicos.

Entre los muchos que se han generado durante el siglo pasado se destacan dos grupos fundamentales: Los índices de diversidad, de los cuales el más utilizado por su eficacia ha sido el índice de Shannon-Weaver (H), generado en 1963, teniendo en cuenta la teoría matemática de la información (Shannon \& Weaver, 1949) y su aplicación en Ecología fue propuesta inicialmente por Margalef (1968). Este índice mide en unidades de bits/individuos con escala logarítmica base 2. En el contexto de los ecosistemas fluviales el índice adquiere un valor máximo de 4,5 bits/individuo para las comunidades de macroinvertebrados bentónicos (Daniel, 2002). Valores inferiores a 2,4-2,5 bits/individuo son indicativos de que el ecosistema se encuentra sometido a tensión (vertidos, dragados, canalizaciones, regulación por embalses, etc.). El índice disminuye mucho en aguas contaminadas. El segundo grupo está constituido por los Índices Bióticos, basados en el nivel de tolerancia de organismos a diferentes grados de contaminación en el agua.

Los dos índices más empleados han sido el Índice Biótico de Hilsenhoff (IB), originalmente desarrollado en la Universidad de Wisconsin-Madison (Hilsenhoff, 1977), para valorar las bajas concentraciones originadas por el vertimiento de materia orgánica en ríos de Estados Unidos. Este índice exige determinaciones sistemáticas hasta el nivel de especies, aunque en los últimos años hay propuestas de su utilización empleando el nivel de familia. El otro es el Biological Monitoring Working Party (BMWP) por sus siglas en inglés, creado en Inglaterra por Hellawell (1986), a diferencia del IB, fue concebido para analizar la tolerancia de familias de 
macroinvertebrados, esto en la práctica resulta ventajoso para aquellos países donde el grado del conocimiento sistemático de los invertebrados es bajo y la diversidad de especies es alta, como en los países neotropicales. El índice fue adaptado por Alba-Tercerdor \& Sánchez Ortega (1988) para la Península Ibérica y fue llamado BMWP.

En Latinoamérica se han realizado algunos intentos de aplicar índices para evaluar la calidad de las aguas. El libro "Bioindicación de la calidad del agua en Colombia" (Roldán, 2002), propone el uso del BMWP/Col. En Costa Rica se hicieron sugerencias para la utilización del BMWP en torrentes tropicales, basado en 40 muestras de cuatro ríos torrentícolas (Hermanson, 1999). En Argentina se investigaron cinco índices bióticos, entre ellos el BMWP, en seis estaciones fluviales, tanto en torrentes montañosos como en la parte baja. En el Suroeste de Polonia, los índices fueron correlacionados con diferentes factores, encontrándose resultados positivos (Chila, 1998). En otro trabajo, realizado también en Argentina, se propone el BMPS (Biotic Monitoring Patagonian Stream) para evaluar la calidad biológica en ríos de la Patagonia. Este índice es una adaptación del BMWP a las condiciones de Argentina (Misrendino \& Pizzolo, 1999). Junquiera et al (2000) realizaron un trabajo relacionado con el BMWP. En el mismo estos autores adaptaron este índice creando tablas de tolerancias de familias a nivel regional para Brasil correlacionando sus resultados con análisis físico-químicos, lo cual reafirmó la eficacia del método. Naranjo et al (2003) crearon el BMWP-Cuba para Cuba, el cual como los anteriores resulta de una adaptación del BMWP. Este índice fue probado en varios ríos del Oriente y Centro del país, dando magníficos resultados.

Es importante mencionar que Honduras, por su topografía, posee muchas cuencas hidrográficas asociadas con ríos, quebradas, lagunas, un lago natural de gran tamaño y varios embalses. Sin embargo se sabe muy poco sobre el grado de contaminación de las mismas.

El desarrollo y dispersión de la población humana en nuestro país junto con la creciente demanda de agua de buena calidad, obliga a los diferentes sectores de la sociedad a realizar esfuerzos para determinar la calidad, localización y disponibilidad de las fuentes de agua para garantizar el uso sostenible de los recursos hídricos existentes para suplir los requerimientos de nuestra sociedad. Pero al igual que muchos otros países en desarrollo, no poseemos los recursos necesarios para realizar monitoreos sistemáticos en todas las localidades donde se requieren. Sin embargo, las experiencias de otros países de condiciones similares a las nuestras y el basamento técnico-científico moderno existente relacionado con el biomonitoreo de cuerpos de agua utilizando los índices bióticos, nos proveen la 
oportunidad para utilizar esos recursos en la solución de la problemática nacional en lo referente a la determinación de la calidad de los recursos hídricos con el objeto de garantizar, hasta donde sea posible, la utilización segura de los mismos para satisfacer las necesidades básicas de nuestra sociedad.

En Honduras existen antecedentes sobre la aplicación de índices bióticos considerando el uso de macroinvertebrados bentónicos a nivel de familia para evaluar la calidad de ciertos cuerpos de agua lóticos. En este sentido se pueden mencionar entre otros; el de Borjas (1984) sobre la calidad del agua del río Chiquito y el de Borjas, et. Al. (1987), sobre río Choluteca. El uso de índices bióticos en la cuenca del río Choluteca para determinar la calidad del agua relacionada con parámetros fisicoquímicos fue realizado por profesionales trabajando para el Laboratorio de Limnología OEA-UNAH en 1992. Borjas et. Al. (1997), realiza una evaluación de la calidad del agua del Parque Nacional Cerro Azul Meámbar en cuanto a la contaminación orgánica y la producida por plaguicidas. Un estudio similar al anterior por Castillo (2002), referente a la distribución de macroinvertebrados y su utilización como indicadores de contaminación orgánica en la cuenca del río Sabacuante. El Centro de Biodiversidad El Zamorano ha realizado algunos trabajos relacionados con Indicadores biológicos en microcuencas aledañas al Parque Nacional La Tigra, en Francisco Morazán, Honduras (PNLT).

En años anteriores, como parte de las actividades de la cátedra de Entomología Acuática y Manejo de Cuencas de la carrera de Biología, actualmente en la Escuela de Biología de la UNAH se han realizado diferentes estudios en la zona de Francisco Morazán y principalmente en el área del Parque Nacional La Tigra, y sus zonas aledañas entre los cuales podemos mencionar algunos: "Diversidad de insectos acuáticos en la quebrada Las Trojas", Gamero y Talavera (2003); "Diversidad de insectos acuáticos en las confluencias de la microcuenca del río San Juan: quebrada Las Trojas, quebrada Las Cañas y quebrada Dulce", Argueta et al (2008); "Insectos acuáticos asociados al río La Fortuna y quebradas aledañas", Aguilar, Meza y Álvarez (2004); "Diversidad de insectos acuáticos y calidad del agua de la quebrada Munuare en Tatumbla, Francisco Morazán", realizado por Montes et al. (2001); "Estudio de la calidad del agua de la quebrada La Salada, La Montañita", Mérida (1996); "Determinación de la calidad del agua de la quebrada La Salada en La Montañita" por Marroquín, Padilla y Medrano (2000); "Evaluación biológica de la microcuenca San Francisco, Valle de Ángeles, Francisco Morazán" Núñez, (2007).

La Fundación Amigos de La Tigra (Amitigra), encargada del manejo y protección del 
Parque Nacional La Tigra, está tratando de monitorear la calidad del agua de las diferentes microcuencas del parque. Dentro del Parque Nacional La Tigra existen varios riachuelos y quebradas destacándose por el lado de San Juancito, el río Chiquito, quebrada Las Trojas y Tata Justo, al suroeste la quebrada Las Cañas, quebrada Dulce y La Jardinera y al sur la quebrada de Santa Elena, quebrada Honda, La Danta, río El Carrizal y la de Agua Dulce (Ecología y Servicios, 1998).

En el período comprendido entre febrero y marzo (época seca) del 2009, los estudiantes de la asignatura de Entomología Acuática de la UNAH, con la colaboración de Amitigra, realizaron un estudio preliminar de la calidad del agua del río Jutiapa en las confluencias de la quebrada Corralitos y quebrada Limones utilizando los insectos acuáticos como bioindicadores.

La falta de información de la entomofauna acuática existente en el área de estudio, obligó a los encargados del trabajo de investigación a realizar un estudio para evaluar la entomofauna acuática como indicadores biológicos de la calidad del agua del río Jutiapa en las confluencias de la quebrada Corralitos y quebrada Limones, en el Parque Nacional La Tigra, departamento de Francisco Morazán.

El objetivo de este estudio fue determinar la abundancia y la diversidad de insectos acuáticos bentónicos como bioindicadores de la calidad del agua y establecer las posibles relaciones con los parámetros físico-químicos de las quebradas antes mencionadas, mediante el análisis de parámetros comunitarios (Índice de Diversidad Shannon-Wienner, Índice de Berger-Parker, Índice de Similitud de Jaccard de la macrofauna bentónica y la aplicación del Índice Biótico de Familias BMWP-CR para determinar la calidad del agua de las quebradas seleccionadas que permitan satisfacer la información para el uso del recurso.

\section{MATERIALES Y MÉTODOS}

\section{Área de Estudio}

El río Jutiapa se encuentra ubicado en la parte NE del PNLT, pasando por diferentes comunidades como ser: Casa Quemada, Jutiapa arriba, Jutiapa abajo, Limones y Corralitos en El Hatillo, zona de amortiguamiento del Parque. Ubicado a 11 kilómetros al NE de la ciudad de Tegucigalpa, FM, Honduras.

El río Jutiapa tiene un área total de $45.30 \mathrm{~km}$ cuadrados, un perímetro de $36.9 \mathrm{kms}$, la longitud del cauce principal de $5.83 \mathrm{kms}$. La altura máxima de $2140 \mathrm{msnm}$ y la altura mínima $900 \mathrm{msnm}$. La distancia de alturas en línea recta de $10.212 \mathrm{~km}$. Es un 
río de orden 3 con el método Strahler, tiene una densidad de drenaje de $0.55 \mathrm{~km}$ y una constante de mantenimiento de 1.81. El Coeficiente de Gravellius es de 1.54, de forma oblonga, con una pendiente de la cuenca de $0.01 \%$ y la pendiente del cauce principal de $21.2 \%$, el tiempo de concentración de $0.20 \mathrm{hrs}$., el alejamiento medio 0.86 , el Índice de Alivio de 0.12 y la frecuencia de $0.28 \mathrm{~km}$ cuadrados y un patrón de drenaje dendrítico (Anexo 1).
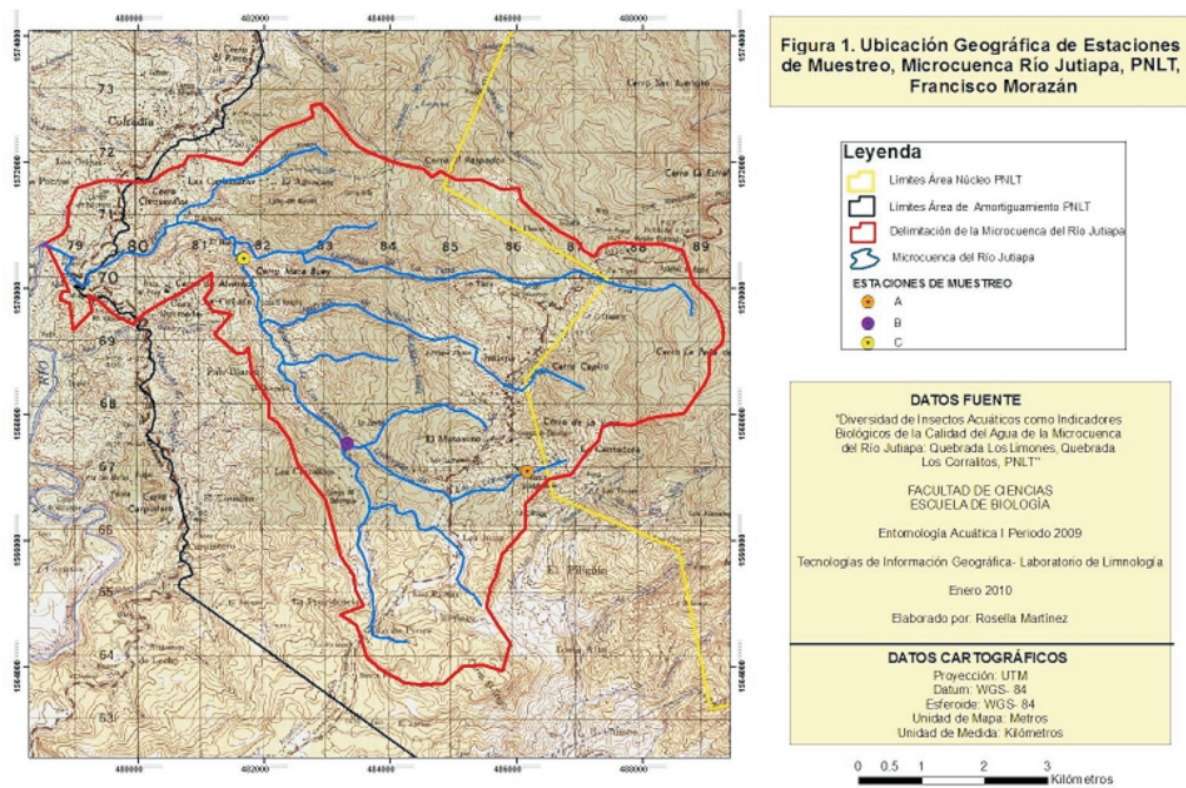

Figura 1. Ubicación geográfica de las estaciones de muestreo en las microcuencas del río Jutiapa, Parque Nacional La Tigra, Francisco Morazán (PNLT).

Para realizar el presente estudio en el curso del río Jutiapa, se seleccionaron las microcuencas correspondientes a la quebrada Limones y la quebrada Corralitos y el inicio del río Jutiapa (Fig. 1).

\section{Descripción de las quebradas estudiadas}

\section{Quebrada Limones (Fig. 2)}

La quebrada Limones está ubicada en la zona de amortiguamiento del PNLT, cerca de la comunidad de Limones en la parte sur y en la parte norte con la comunidad de Jutiapa abajo, en las coordenadas 16486064 Este 1567324 Norte y con una elevación de 1484 msnm. 
La quebrada recorre varias zonas de cultivo y el agua es utilizada para irrigación. Esta zona presenta una vegetación predominante de gramíneas y hierbas con muy pocos árboles. Las especies de árboles encontradas son: liquidámbar y pino en la parte alta.

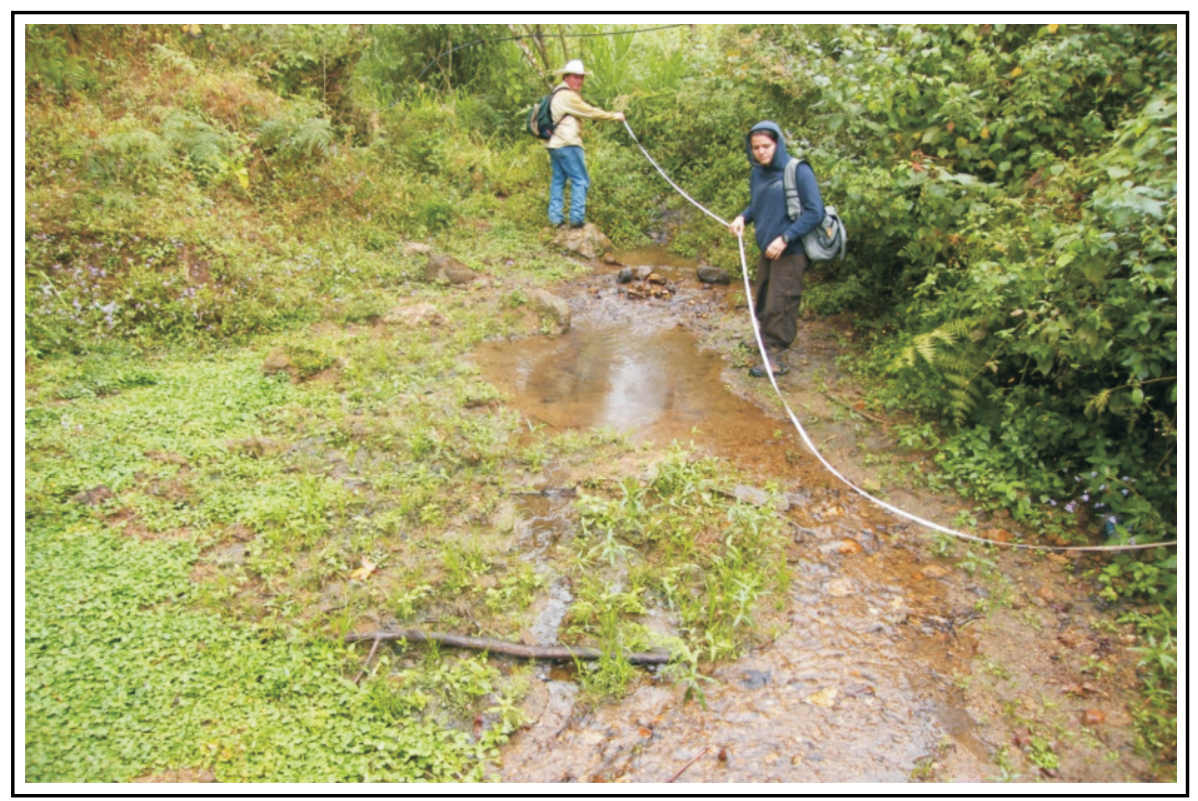

Figura 2. Quebrada Limones

En esta quebrada se observó un caudal muy bajo al momento de realizar el estudio, como se observa en la figura 2 con un lecho arenoso y con un flujo muy lento.

\section{Quebrada Corralitos (Figuras 3y 4 )}

La quebrada Corralitos, en la zona de amortiguamiento del PNLT, se encuentra ubicada en la zona de El Hatillo, con las coordenadas 16483349 Este y 1567494 Norte a una elevación de $1252 \mathrm{msnm}$.

En las orillas de esta quebrada se encuentra una finca de café cuyas aguas mieles y otros desperdicios son vertidos directamente en la quebrada (fotografía de la derecha).

El estudio se realizó en la intersección con la quebrada Limones menos contaminada que la de Corralitos, como se puede observar en la fotografía de la izquierda. 


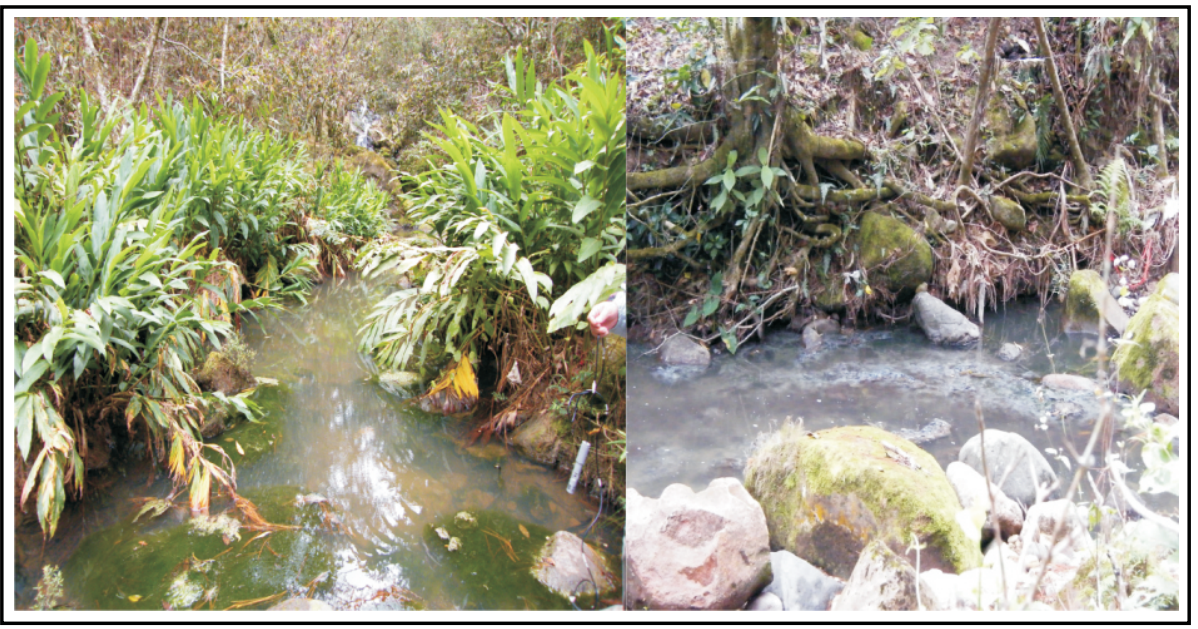

Figura 3. La imagen de la izquierda, quebrada Limones y la imagen de la derecha, quebrada Corralitos.

La vegetación de las riberas está formada predominantemente por Heliconiáceas, árboles de Quercus y muchos árboles de manzanitas.

El lecho del río es rocoso y el flujo lento con agua lechosa, estancada, con mal olor por contaminación orgánica (Fermentación de pulpa de café) como se observa en las figuras 3 y 4.

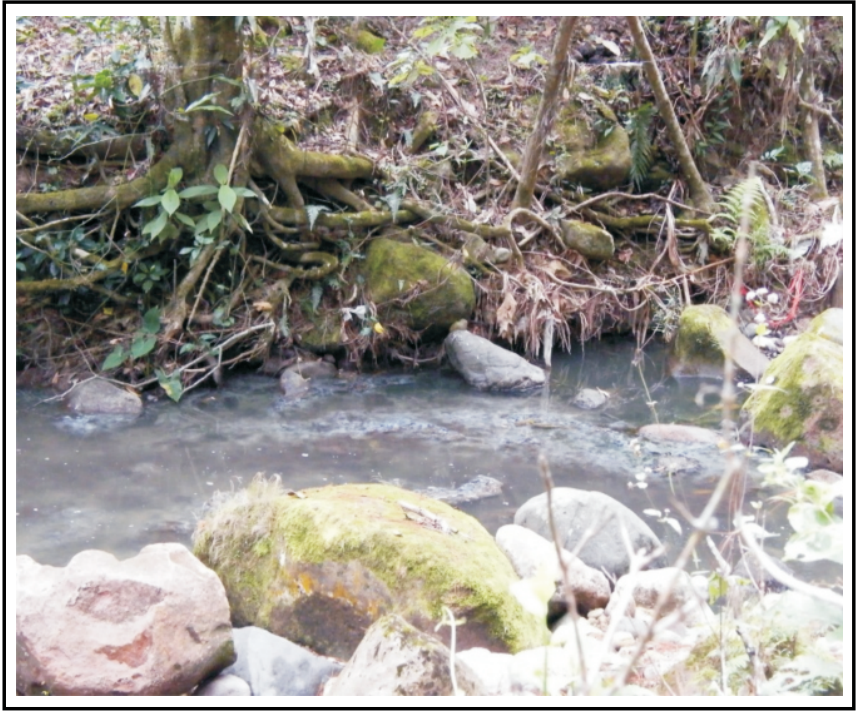

Figura 4. Quebrada Corralitos.

36 Dirección de Investigación Científica 


\section{Inicio del río Jutiapa. (Figuras 5 y 6 )}

El inicio del río Jutiapa $(C)$ al lado NE del PNLT cerca de las comunidades de Carpinteros y Casa Quemada, y al Este del PNLT conocida como La Tigrita o Tigra Abajo. Las coordenadas son 16481726 Este y 1570431 Norte.

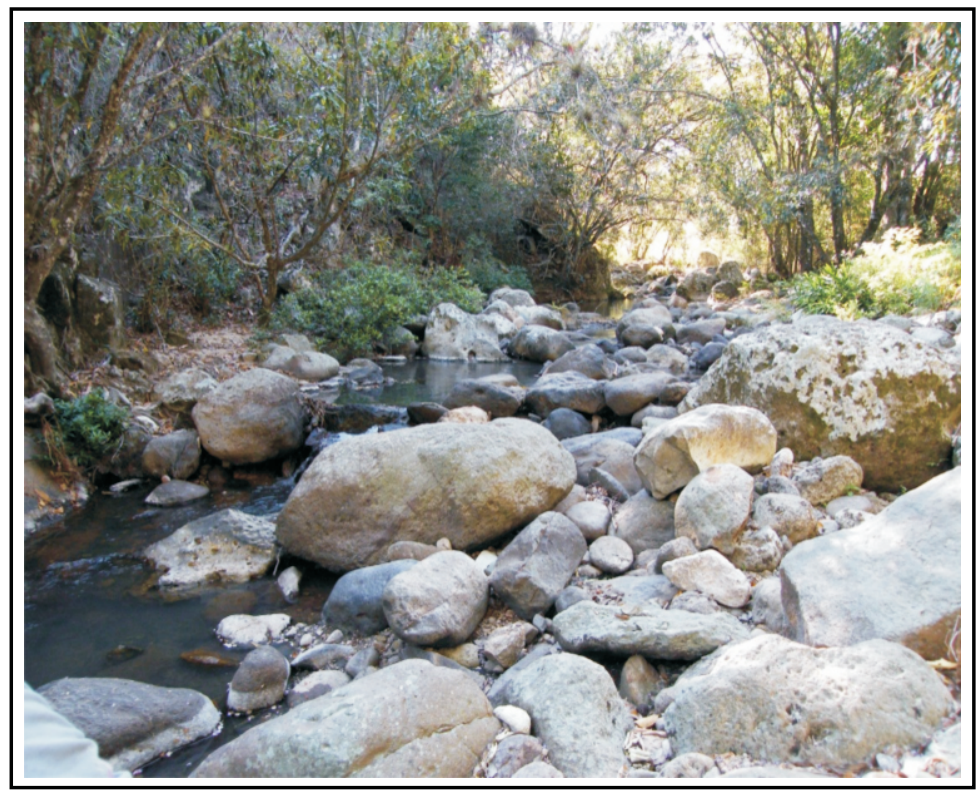

Figura 5. Río Jutiapa (C)

El área de estudio del río Jutiapa (Fig. 5) está caracterizada por un lecho pedregoso en la mayoría de su trayectoria, aunque en algunas zonas se pudo observar un lecho arenoso.

Debido al hecho que existían varias pozas que se forman entre las rocas, no se pudo medir la velocidad de la corriente, la profundidad y la anchura del mismo cuando el flujo de agua era bajo.

La vegetación de la microcuenca está constituida especialmente por pinos y en algunas zonas se pueden observar árboles Quercus sp. y en las zonas de mayor elevación Liquidámbarsp..

En las riberas del río se realiza actividad agrícola y ganadera. En el trayecto se observó un cultivo de maíz, combinado con algunas cucurbitáceas (Fig. 6), así como actividad de preparación de tierras para cultivar. 


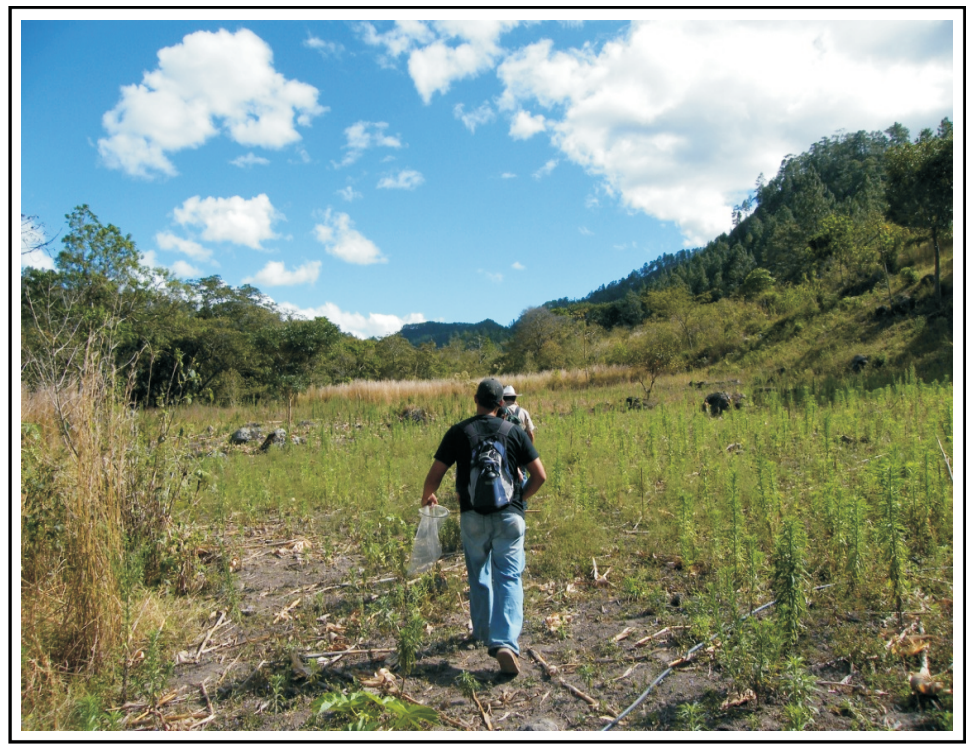

Figura 6. Área de cultivo cerca del río Jutiapa.

\section{Métodos de campo y laboratorio}

Con el fin de estudiar la abundancia y la diversidad de insectos acuáticos bentónicos como bioindicadores de la calidad del agua y establecer las posibles relaciones con los parámetros físico-químicos de la microcuenca: río Jutiapa en la quebrada Corralitos y quebrada Limones en el Parque Nacional La Tigra, Francisco Morazán, se realizaron dos muestreos durante la época seca entre el 28 de febrero y el 21 de marzo del 2009, conjuntamente se midieron de manera puntual algunas variables ambientales, morfométricas y físico-químicas en las 3 quebradas mencionadas anteriormente.

La ubicación de las estaciones para monitorear los insectos acuáticos y los parámetros físico-químicos fue definida según representatividad del área en términos de distancia e influencia de actividad antropogénica y por la trama de caminos rurales que facilitaron el acceso a los puntos de muestreo.

En cada quebrada, en una sección de aproximadamente 2 metros lineales se colectaron los insectos acuáticos, utilizando pinzas y pinceles en 3 hábitats diferentes escogidos al azar (debajo de las piedras, en substrato arenoso-limoso y en las hojarascas), durante 25 minutos. Se seleccionaron las confluencias de las quebradas para realizar las colectas, obteniéndose así 3 muestras por quebrada 
para cada fecha de muestreo. Así mismo, se ubicaron las coordenadas con el GPS y la altitud utilizando un altímetro. Se procedió a la medida de variables ambientales como ser la temperatura del aire y la de la superficie del agua, con un termómetro de mercurio.

La conductividad específica en $(\mu \mathrm{h})$ por su parte, fue medida con un conductímetro (Modelo DA-1) Lamotte. Se midió la profundidad (m), el ancho $(\mathrm{m})$, la velocidad de la corriente $(\mathrm{m} / \mathrm{s})$, y por último la descarga del río (metros cúbicos por segundo mediante el método del flotador) (Allan 1995).

Los parámetros químicos monitoreados fueron el pH del agua mediante el uso de cinta reactiva de $\mathrm{pH}$, el oxígeno disuelto (mg/l) mediante un oxinómetro (YSI Modelo 57), y la prueba de la DBO5. Los insectos colectados en cada muestreo fueron colocados en viales y preservados en alcohol al $70 \%$ para posteriormente ser trasladados al laboratorio de aguas de la Escuela de Biología de la UNAH. En el laboratorio, los insectos fueron limpiados de detritus, separados por orden, contados y posteriormente colocados en alcohol limpio, para su respectiva clasificación. Los insectos acuáticos colectados fueron identificados con ayuda de claves taxonómicas (Merrit \& Cummins, 1996) en órdenes, familias y géneros.

Los ejemplares colectados e identificados fueron catalogados como parte de la colección de referencia de insectos acuáticos de la Escuela de Biología de la UNAH.

\section{Métodos de análisis de datos}

Con los datos obtenidos se compararon las 3 quebradas de la microcuenca Jutiapa (río Jutiapa (C) en la quebrada Corralitos (B) y quebrada Limones (A) ) utilizando:

El índice de similitud de Jaccard $=(\mathrm{J} / \mathrm{r} \times 100)$, basado en la presencia o ausencia de familias. En donde $\mathrm{J}$ significa el número de especies en común en las tres quebradas y r la suma de las especies exclusivas de a y b.

El índice de Berger y Parker (B) que mide la dominancia de la especie o taxón más abundante, siendo su expresión matemática $\mathrm{B}=\mathrm{N} \max / \mathrm{N}$, en donde Nmax es el número de individuos en el taxón más abundante y $\mathrm{N}$ es el número total de individuos de la muestra (Soothwood 1978).

El índice de Diversidad de Shannon-Wienner $H^{\prime}=\Sigma p_{i} \mid n p_{i}$, este índice es uno de los índices de diversidad más utilizados para determinar la diversidad de especies de un determinado hábitat. Expresa la uniformidad de los valores de importancia a 
través de todas las especies de la muestra. Además mide el grado promedio de incertidumbre en predecir a qué especie pertenecerá un individuo escogido al azar de una colección (Baev \& Penev, 1995; Maguerra, 1988; Moreno, 2001; Peet, 1974). Asume que los individuos son seleccionados al azar y que todas las especies están representadas en la muestra. Es un índice muy susceptible a la abundancia.

Para determinar la calidad del agua de las quebradas seleccionadas se utilizó el Índice BMWP-CR cuyas siglas en inglés significan Biological Monitoring Working Party modificado para Costa Rica. Este índice se deriva de la cuantificación de presencia y ausencia de las familias de macroinvertebrados identificados con diferente tolerancia a la contaminación (INBio TNC. 2008). Es un índice que se calcula sumando las puntuaciones asignadas a las distintas familias de macroinvertebrados encontrados según su grado de sensibilidad a la contaminación, sin embargo en el caso de nuestro estudio, se trabajó con insectos acuáticos. Es importante mencionar que el puntaje se asigna una sola vez/familia, independientemente de la cantidad de individuos o géneros encontrados.

\section{RESULTADOS}

Los resultados de los parámetros físico-químicos tomados en las diferentes quebradas estudiadas de la microcuenca río Jutiapa se presentan en la tabla 1.

Tabla 1. Parámetros físico-químicos

\begin{tabular}{l|c|c|c|c|c|c|c|}
\hline \multirow{2}{*}{ Parámetro } & \multicolumn{4}{c|}{ Muestreo 1 } & \multicolumn{3}{c}{ Muestreo 2 } \\
\cline { 2 - 8 } & Estación A & Estación B & Estación C & Estación A & Estación B & Estación C \\
\hline Temperatura Ambiente & $30^{\circ} \mathrm{C}$ & $20^{\circ} \mathrm{C}$ & $15^{\circ} \mathrm{C}$ & $17^{\circ} \mathrm{C}$ & $19^{\circ} \mathrm{C}$ & $19.5^{\circ} \mathrm{C}$ \\
\hline Temperatura Agua & $24^{\circ} \mathrm{C}$ & $17^{\circ} \mathrm{C}$ & $12^{\circ} \mathrm{C}$ & $23^{\circ} \mathrm{C}$ & $23^{\circ} \mathrm{C}$ & $26^{\circ} \mathrm{C}$ \\
\hline $\mathrm{pH}$ & 5 & 2 & 7 & 7 & 8 & 6 \\
\hline Conductividad & $150 \mu \Omega$ & $300 \mu \Omega$ & $250 \mu \Omega$ & $300 \mu \Omega$ & $400 \mu \Omega$ & $600 \mu \Omega$ \\
\hline Oxígeno & $3 \mathrm{ppm}$ & $4 \mathrm{ppm}$ & $7 \mathrm{ppm}$ & $8.5 \mathrm{ppm}$ & $4 \mathrm{ppm}$ & $7 \mathrm{ppm}$ \\
\hline $\mathrm{DBO}_{5}$ & $0.4 \mathrm{ppm}$ & $\mathrm{N} . \mathrm{D}$ & $1.5 \mathrm{ppm}$ & $6.1 \mathrm{ppm}$ & $2 \mathrm{ppm}$ & $2 \mathrm{ppm}$ \\
\hline
\end{tabular}

Muestreo 1

\begin{tabular}{|c|c|c|}
\hline \multicolumn{3}{|c|}{ Velocidad de la corriente m/seg } \\
\hline Estación A & Estación B & Estación C \\
\hline 0.212 & ------- & 0.079 \\
\hline
\end{tabular}

Muestreo 2

Velocidad de la corriente $\mathrm{m} / \mathrm{seg}$

\begin{tabular}{|c|c|c|}
\hline Estación A & Estación B & Estación C \\
\hline 0.082 & ------ & ------ \\
\hline
\end{tabular}




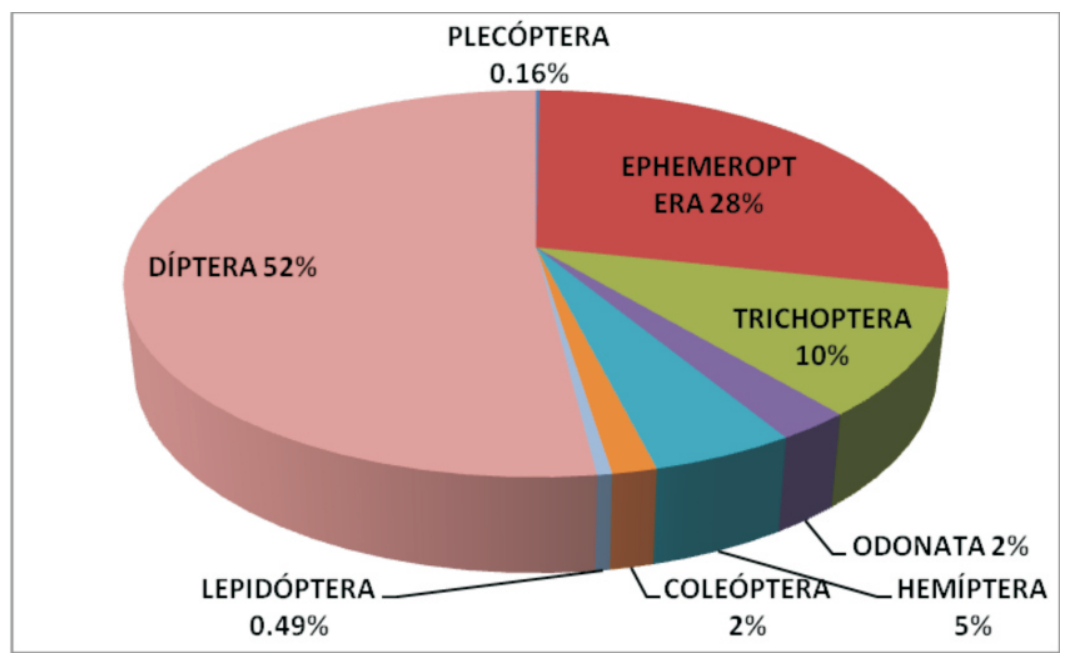

Figura 7. Representación gráfica de los órdenes de los insectos acuáticos colectados durante el estudio en las quebradas Limones, Corralitos y Jutiapa del Parque Nacional La Tigra, Francisco Morazán, Honduras. Época seca del 2009.

En este estudio, realizado durante los meses de febrero y marzo (época seca) del 2009 en la quebrada Limones, quebrada Corralitos y quebrada Jutiapa, se colectó un total de 612 individuos pertenecientes a 8 órdenes de insectos. Se registró un total de 27 familias, la mayoría de las cuales (75\% del total) corresponden a estados inmaduros de insectos.

Los órdenes más abundantes fueron: el orden Díptera con 318 individuos que representan el $51.96 \%$ del total de individuos colectados; el orden Ephemeroptera con 173 individuos (28.27\%); el orden Trichoptera con 64 (10.46\%); el orden Hemíptera con 29 individuos (4.74\%); el orden Odonata con 15 ejemplares (2.45\%); el orden Coleóptera con 9 ejemplares (1.47\%); el orden Lepidóptera con 3 individuos $(0.495 \%)$ y el orden Plecóptera con 1 individuo (0.16\%). Información contenida en la Fig. 7 y Tabla 2.

De los órdenes encontrados en todo el estudio, el orden que presentó más familias fue el orden Díptera con 6 familias. En segundo lugar le sigue el orden Ephemeroptera con 4 familias, el orden Trichoptera con 4 familias, el orden Hemíptera, el orden Coleóptera y el orden Odonata con 3 familias, cada uno; el orden Plecóptera y el orden Lepidóptera con 1 familia respectivamente, como se muestra en la Tabla 2. 


\section{Composición, abundancia y riqueza de familias}

Las familias que presentaron mayor abundancia durante el estudio, como se muestra en la Tabla 2 y Fig. 8, fueron: La familia Culicidae con 194 individuos que le corresponde el 31.6\%, la familia Simulidae con 106 individuos (17.3\%), la familia Leptophlebiidae con 115 individuos (18.79\%), Hydropsichidae 59 individuos (9.6\%) y la familia Baetidae con 50 individuos (8.16\%). La abundancia de las demás familias, como se muestra en la Tabla 2 y en la Fig. 8 , es poco significativa.

La abundancia de individuos por quebrada resultó ser mayor en la quebrada Corralitos con 371 individuos, le sigue la quebrada Limones con 177 individuos y en tercer lugar la quebrada Jutiapa con 64 individuos como se muestra en la Tabla 2.

Tabla 2. Resumen de los resultados obtenidos en la clasificación de la calidad del agua en las confluencias del río Jutiapa: quebrada Limones, quebrada Corralitos y quebrada Jutiapa. Época seca del 2009

\begin{tabular}{|c|c|c|c|c|c|c|c|}
\hline \multirow{2}{*}{ ORDEN } & \multirow{2}{*}{ FAMILIA } & \multicolumn{2}{|c|}{$\begin{array}{c}\text { Quebrada Limones } \\
\text { A }\end{array}$} & \multicolumn{2}{|c|}{$\begin{array}{c}\text { Quebrada Limones } \\
\text { B }\end{array}$} & \multicolumn{2}{|c|}{$\begin{array}{c}\text { Quebrada Limones } \\
\text { C }\end{array}$} \\
\hline & & BMW P-CR & $\begin{array}{c}\text { No. de } \\
\text { individuos }\end{array}$ & BMW P-CR & $\begin{array}{c}\text { No. de } \\
\text { individuos }\end{array}$ & BMW P-CR & $\begin{array}{c}\text { No. de } \\
\text { individuos }\end{array}$ \\
\hline PLECÓPTERA & Perlidae & 10 & 1 & & 0 & & 0 \\
\hline \multirow{4}{*}{ EPHEMEROPTERA } & Baetidae & 5 & 16 & 5 & 30 & 5 & 4 \\
\hline & Baeticidae & & 1 & & 0 & & 0 \\
\hline & Leptophlebiidae & 8 & 64 & 8 & 11 & 8 & 40 \\
\hline & Heptagenidae & & 0 & 10 & 6 & 10 & 1 \\
\hline \multirow{4}{*}{ TRICHOPTERA } & Hydropsichidae & 5 & 47 & 5 & 9 & 5 & 3 \\
\hline & Hydroptilidae & & 0 & 6 & 1 & & 0 \\
\hline & Psichomyiidae & & 0 & & 1 & & 0 \\
\hline & Glossomatide & 8 & 3 & & 0 & & 0 \\
\hline \multirow{3}{*}{ ODONATA } & Coenagrionidae & 5 & 3 & 5 & 1 & 4 & 4 \\
\hline & Calopterigidae & 5 & 4 & 5 & 1 & 4 & 1 \\
\hline & Libelulidae & & 0 & & 0 & 6 & 1 \\
\hline \multirow{4}{*}{ HEMIPTERA } & Veliidae & & 13 & & 2 & & 0 \\
\hline & Guerridae & & 4 & & 7 & & 0 \\
\hline & Notonectidae & 4 & 2 & & 0 & & 0 \\
\hline & Naucoridae & & 0 & 4 & 1 & & 0 \\
\hline \multirow{3}{*}{ COLEÓPTERA } & Psephenidae & 7 & 3 & & 0 & 7 & 1 \\
\hline & Staphilinidae & 4 & 1 & 4 & 2 & & 0 \\
\hline & Elmidae & 5 & 1 & 5 & 1 & & 0 \\
\hline LEPIDÓPTERA & Piralidae & 5 & 1 & & 0 & 5 & 2 \\
\hline \multirow{7}{*}{ DIPTTERA } & Culicidae & & 0 & 2 & 194 & & 0 \\
\hline & Tipulidae & & 0 & 4 & 1 & 4 & 2 \\
\hline & Adulto N.D. & & 0 & & 1 & & 0 \\
\hline & Simulidae & 4 & 12 & 4 & 89 & 4 & 5 \\
\hline & Ceratopognidae & 4 & 1 & & 0 & & 0 \\
\hline & Chaoboridae & & 0 & & 4 & & 0 \\
\hline & Chironomidae & & 0 & 2 & 9 & & 0 \\
\hline \multicolumn{2}{|c|}{ TOTAL } & 79 & 177 & 69 & 371 & 62 & 64 \\
\hline
\end{tabular}


Tabla 3. Resumen de los resultados de los diferentes parámetros comunitarios calculados para río Jutiapa: quebrada Limones, quebrada Corralitos y quebrada Jutiapa durante la época seca del 2009

\begin{tabular}{|c|c|c|c|}
\hline & $\begin{array}{c}\text { Quebrada Limones } \\
\text { A }\end{array}$ & $\begin{array}{c}\text { Quebrada Corralitos } \\
\text { B }\end{array}$ & $\begin{array}{c}\text { Quebrada Jutiapa } \\
\text { C }\end{array}$ \\
\hline $\begin{array}{c}\text { CALIDAD DEL AGUA } \\
\text { BMW P-CR }\end{array}$ & $\begin{array}{l}\text { AGUAS DE CALIDAD } \\
\text { REGULAR, EUTROFIA } \\
\text { CONTAMINACIÓN } \\
\text { MODERADA }\end{array}$ & $\begin{array}{c}\text { AGUAS DE CALIDAD } \\
\text { REGULAR, EUTROFIA } \\
\text { CONTAMINACIÓN } \\
\text { MODERADA }\end{array}$ & $\begin{array}{l}\text { AGUAS DE CALIDAD } \\
\text { REGULAR, EUTROFIA } \\
\text { CONTAMINACIÓN } \\
\text { MODERADA }\end{array}$ \\
\hline $\begin{array}{l}\text { ÍNDICE DE DIVERSIDAD DE } \\
\text { SHANNON-WIENNER }\end{array}$ & 1.91618219 & 1.5439497 & 1.45949014 \\
\hline $\begin{array}{l}\text { ÍNDICE DE DOMINANCIA DE } \\
\text { BERGER-PARKER }\end{array}$ & $\begin{array}{c}0.056497175 \\
\text { Leptophlebiidae }\end{array}$ & $\begin{array}{l}0.522911051 \\
\text { Culicodae }\end{array}$ & $\begin{array}{c}0.625 \\
\text { Leptophlebiidae }\end{array}$ \\
\hline
\end{tabular}

Índice de similitud de Jaccard (Estación A y B)

Índice de similitud de Jaccard (Estación B y C)

Índice de similitud de Jaccard (Estación A y C)

$62.5 \%$

$57.1 \%$

$66.6 \%$

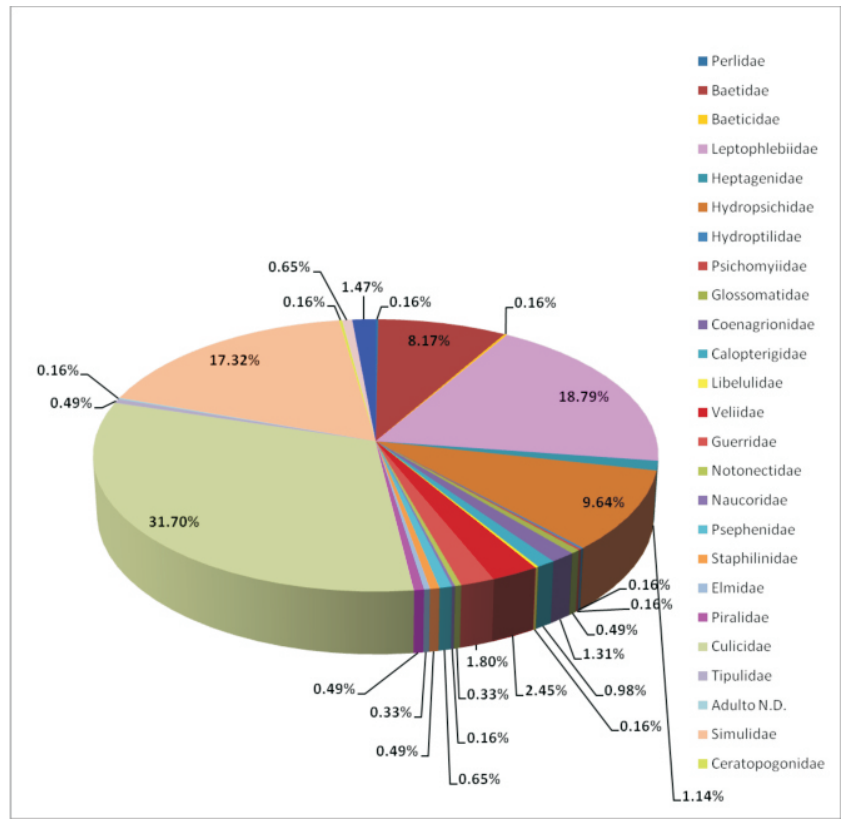

Figura 8. Representación gráfica de las familias de insectos acuáticos colectados durante el estudio en las quebradas Limones, Corralitos y Jutiapa del Parque Nacional La Tigra, Francisco Morazán, Honduras. Época seca del 2009. 
Composición de la riqueza específica y dominancia de familias por quebrada.

De acuerdo a los resultados obtenidos al calcular el índice de diversidad de Shannon-Wiener $\left(H^{\prime}\right)$ que aparecen en la Fig. 9 y Tabla 3, el valor para la quebrada Limones (A) fue de 1,001577851; para la quebrada Corralitos (B) un índice de 0,670528836 y el de la quebrada Jutiapa (C) fue de 0,617957163.

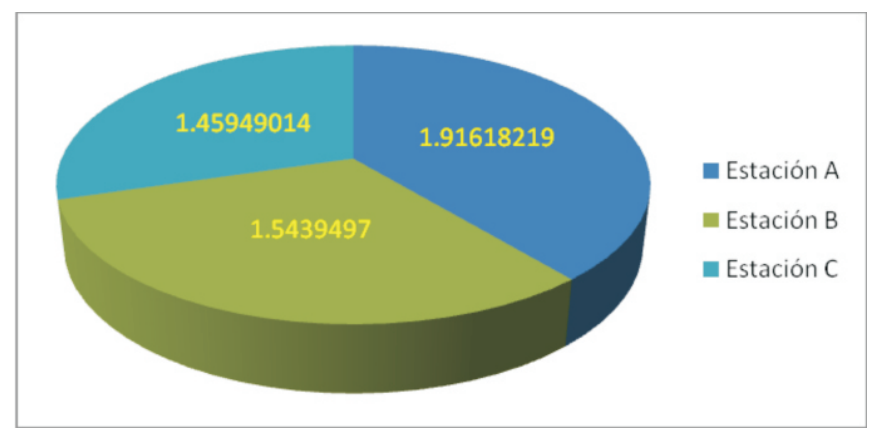

Figura 9. Representación gráfica del Índice de Diversidad de Shannon-Wienner obtenido en el estudio de calidad de agua en las quebradas Limones, Corralitos y Jutiapa del Parque Nacional La Tigra, Francisco Morazán, Honduras, en la época seca del 2009.

Los resultados del índice de Dominancia de Berger-Parker demuestran un valor de 0.0564977851 para la quebrada Limones (A), dominando la Familia Leptophlebiidae. Un índice de 0,522911051 para la quebrada Corralitos (B), dominando la familia Culicidae, y la quebrada Jutiapa (C) presentó un índice de Dominancia de 0,625, dominando la familia Leptophlebiidae (Fig. 10 y Tabla 3 ).

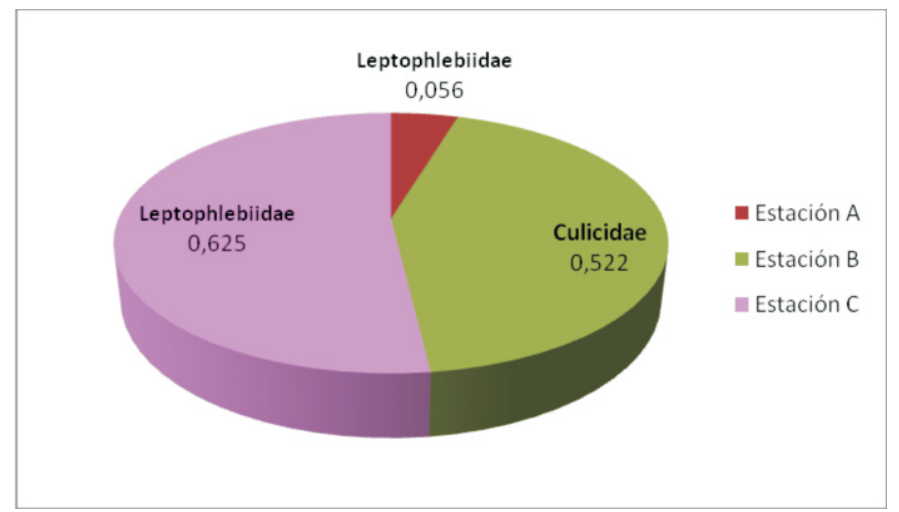

Figura 10. Representación gráfica del Índice de Dominancia de Berger-Parker obtenido en el estudio de calidad de agua en las quebradas Limones, Corralitos y Jutiapa del Parque Nacional La Tigra, Francisco Morazán, Honduras. Época seca del 2009. 
El índice de Similitud de Jaccard que demuestra la similitud y la disimilitud entre las áreas muestreadas (Quebradas: Limones A, Corralitos B y Jutiapa C), y nos dice de acuerdo al resultado cuál es la heterogeneidad biótica de la comunidad. De acuerdo a los resultados podemos determinar qué similaridad existe entre quebradas.

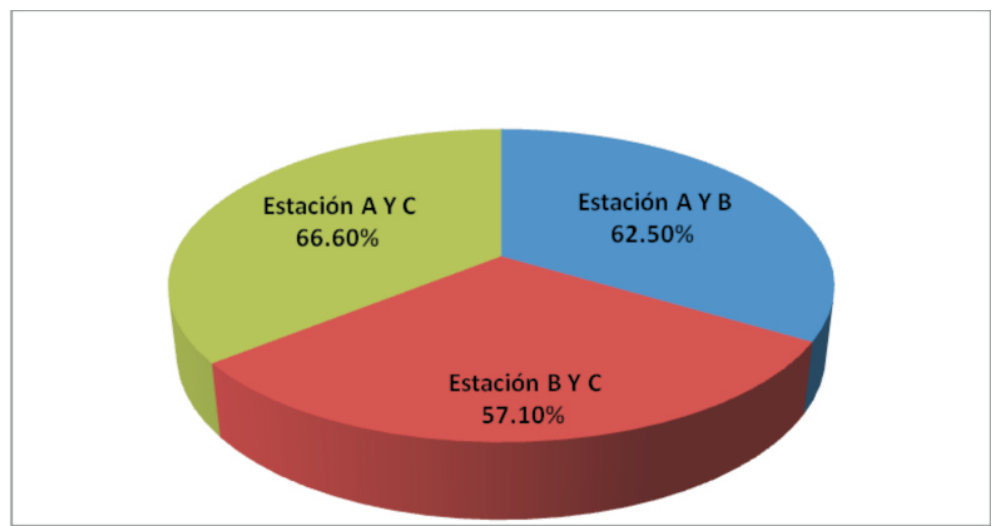

Figura 11. Representación gráfica del Índice de Similitud de Jaccard obtenido en el estudio de calidad de agua en las quebradas Limones, Corralitos y Jutiapa del Parque Nacional La Tigra, Francisco Morazán, Honduras. Época seca del 2009.

En la Fig.11, Tabla 3, se puede observar que el índice de similitud entre la quebrada Limones y la quebrada Corralitos (A y B) fue de $62.5 \%$; el de la quebrada Limones y la quebrada Jutiapa (B y C) fue de $57.1 \%$ y en lo que respecta a la quebrada Limones y la quebrada Jutiapa (Ay C) el índice de similitud fue de $66.6 \%$.

El Índice Biótico de Calidad del Agua BMWP Modificado para Costa Rica, BMWP-CR (In Bio-TNC, 2008), el análisis de la calidad de agua de las quebradas antes mencionadas, que considera la diversidad de taxa (familias indicadoras) y la abundancia de cada una de ellas, es presentada en la Tabla 2. Los resultados con la aplicación del BMWP-CR muestran valores relacionados con la presencia y ausencia de las familias de insectos acuáticos que poseen diferente tolerancia a la contaminación, dependiendo de la sensibilidad.

El índice Biótico de la quebrada Limones fue de 79; el de la quebrada Corralitos fue de 69 y el de la quebrada Jutiapa fue de 62, todas incluidas en el mismo nivel de calidad de agua (ver anexo 1). 


\section{DISCUSIÓN Y CONCLUSIONES}

No existe información específica que guarde relación a estudios que caractericen la macrofauna bentónica del río Jutiapa; ni de información de parámetros fisicoquímicos.

Las investigaciones previas relacionadas con el estudio de diversidad de macroinvertebrados bentónicos 0 de insectos acuáticos como indicadores biológicos, han sido realizadas en otras microcuencas en zonas aledañas al PNLT, como el realizado por Gamero y Talavera, 2003 en la quebrada Las Trojas. El realizado por Argueta et al, 2008, en las confluencias de la microcuenca del río San Juan en las quebradas Las Trojas, Las Cañas y quebrada Dulce, así como la evaluación biológica de la microcuenca San Francisco en Valle de Ángeles por Núñez, 2007.

Todos estos estudios describen la condición ambiental de las microcuencas a través de parámetros físicos y químicos e indicadores biológicos, estudios de abundancia y de diversidad de insectos acuáticos. Las estaciones que fueron seleccionadas para los muestreos presentan diversidad considerable y las tres poseen características completamente diferentes en cuanto a la temperatura, conductividad, tipo de fondo, velocidad de la corriente, anchura, tipo de substrato, oxígeno disuelto, demanda bioquímica de oxígeno, vegetación, tipo de hábitats y microhábitats, así como por las intervenciones humanas, entre otras cosas.

En condiciones naturales la diversidad y la distribución de los insectos acuáticos está limitada por factores como la luz, temperatura, sustrato, ancho del río, velocidad de la corriente, factores de altitud, alimento, hábitat y química del agua (Medianero y Samaniego, 2004).

De acuerdo a los datos morfométricos, la microcuenca del río Jutiapa es ovalada, característica que es muy importante ya que da lugar a una mayor respuesta del cauce ante un evento de crecida, así como la pendiente del cauce principal que es favorable para que el agua escurra rápidamente a ese mismo evento. De acuerdo a la densidad de drenaje de la microcuenca, los volúmenes de agua que escurren durante la época lluviosa son grandes, aunque durante la realización de este estudio todas las quebradas mostraron poco flujo, poca anchura y poca profundidad, sobre todo la quebrada Limones y quebrada Jutiapa en donde fue posible medir la velocidad de la corriente. Los resultados de los parámetros físicoquímicos (Tabla 1) muestran variaciones considerables en ambos muestreos, aquí pudo haber influido la hora del día ya que los muestreos se realizaron a la inversa. 
La conductividad resultó muy alta en los dos muestreos y para las 3 quebradas, esto pudo deberse al arrastre de sedimentos producto de la erosión de áreas adyacentes. En el arrastre de sedimentos van muchos iones disueltos, lo que da como resultado una conductividad específica alta.

El pH resultó significativamente ácido durante el primer muestreo para la quebrada Limones $(A)$, y muy ácido para quebrada Corralitos $(B)$, lo que coincide con el grado de contaminación orgánica producida por aguas mieles. De igual forma el oxígeno disuelto resultó muy bajo para quebrada Limones solamente durante el primer muestreo, esto pudo deberse a la dificultad de medición por la poca profundidad, y el sedimento limoso del lecho de la quebrada. Para la quebrada Corralitos el oxígeno disuelto fue bajo durante los dos muestreos. Los resultados de la $\mathrm{DBO}_{5}$ resultaron significativamente altos para la quebrada Jutiapa durante el segundo muestreo, esto pudo deberse a la cantidad de materia orgánica en descomposición. Este resultado no es congruente con los valores de oxígeno disuelto durante este mismo muestreo, sin embargo, esto pudo deberse a que las aguas corren considerablemente, dando como resultado una buena oxigenación.

En la quebrada Jutiapa se pudo observar la extracción de agua para riego de cultivos y consumo humano de la población adyacente a través de mangueras y aproximadamente a 4 metros del lugar muestreado se observó una pequeña represa en la que se cultivan peces dentro de una propiedad privada.

La quebrada Corralitos presentó considerable anchura (5 metros aproximadamente), pero no fue posible la medición de velocidad de la corriente, ni de profundidad en ninguno de los dos muestreos debido al tipo de lecho del río que es sumamente rocoso y la lentitud del flujo de agua a causa de la contaminación por aguas mieles por los desechos de café. El lecho rocoso para quebrada Corralitos y quebrada Jutiapa indican que durante la época lluviosa son grandes volúmenes de agua que pasan por dichas quebradas; lo que ocasiona una gran variación de los parámetros físico-químicos y comunitarios (de los insectos) calculados durante este estudio.

El número de individuos varió durante ambos muestreos, siendo más abundante en el segundo muestreo, pero la diversidad por familia fue mayor durante el primer muestreo, estas diferencias fueron el resultado del gran número de individuos de la familia Culicidae encontrados durante el segundo muestreo, es de hacer notar que en muchos de los casos cuando el sistema se ve alterado ya sea por medio de las actividades humanas 0 del ambiente, en esos casos algunas especies se ven 
obligadas a sobrevivir adaptándose o simplemente no toleran los cambios del ambiente y desaparecen. Esto da como resultado la pérdida de algunos grupos y el aumento de otros ya sea por la ausencia de depredadores o por la disminución de la competencia de algún nicho específico, por el alimento presente 0 por la combinación de varios factores, como se puede observar en los resultados, el gran número de individuos de la familia Culicidae y Simulidae, comparado con el mínimo número de otras familias presentes en las estaciones monitoreadas.

Los resultados obtenidos en este estudio detectan la presencia de algunos organismos indicadores de aguas relativamente limpias pertenecientes a los ordenes Plecóptera (solamente en la quebrada Limones (A) con la presencia de un organismo de la familia Perlidae), el orden Ephemeroptera (dominando la familia Leptophlebiidae) y del orden Trichoptera (dominando la familia Hydropsychidae) como se puede observar en la Tabla 2. En las quebradas Limones y Jutiapa, en las que resultaron altas concentraciones de oxígeno disuelto y baja demanda bioquímica de oxígeno, el orden Ephemeroptera (familia Leptophlebiidae) fue la más abundante específicamente durante el primer muestreo cuando todavía no se presentaba la época más seca de la estación. La presencia de las órdenes apuntadas anteriormente, por lo general indican aguas de buena calidad.

Sin embargo, en la quebrada Corralitos (B), la mayor abundancia fue para el orden Díptera, específicamente la familia Culicidae y Simulidae (Tabla 2), las cuales agrupan a individuos presentes en casi todos los tipos de ecosistemas loticos y/o lenticos bajo diferentes condiciones ambientales y los cuales han sido reportados como tolerantes a los efectos de la contaminación (Merrit \& Cummins, 1986).

Es de gran importancia recalcar que el agua de la quebrada Corralitos presentó alta contaminación por desechos de café, especialmente aguas mieles, como lo muestran los bajos valores de oxígeno disuelto, alta conductividad, pHácidos, bajo flujo de agua y baja velocidad de la corriente así como la pobreza en la heterogeneidad de los microhábitats. Sin embargo, como lo demuestran los resultados de la Tabla 2, la quebrada Corralitos también presenta abundancia de organismos del orden Ephemeroptera, algunos Trichopteros (Fam. Heptagenidae) y algunos géneros indicadores de aguas limpias como ser organismos del género Choroterpes sp., esto pudo deberse a que muchos de los organismos fueron colectados previos a la confluencia, es decir en la parte baja de quebrada Limones, lo que alteró en gran medida los resultados.

La abundancia de organismos del orden Díptera, con 6 familias presentes y una especie no determinada (ND) son, en su mayoría, indicadoras de contaminación 
del agua. Lo anterior nos permite asegurar que la calidad del agua de la quebrada Corralitos está seriamente dañada.

Sin embargo, es de hacer notar que, en los ríos, la disolución del oxígeno se favorece de forma natural debido a la velocidad de la corriente, pero también se sabe que al aumentar los niveles de materia orgánica debido a descargas de desperdicios, ocurre una disminución natural del oxígeno disuelto y alta demanda bioquímica del oxígeno, que afecta la fisiología de los individuos que componen la comunidad, dando lugar por lo tanto a una abundancia de los organismos más tolerantes a la contaminación y disminuyendo los menos tolerantes. Las órdenes de insectos acuáticos con menos abundancia corresponden a los pertenecientes a los Odonatos, Lepidópteros, Coleópteros y Hemípteros. Es importante mencionar la presencia de la familia Psephenidae del orden Coleóptera (Tabla 2), en las quebradas Limones (A) y Jutiapa $(C)$; que aunque no en abundancia, indica que el agua es de buena calidad.

La diversidad biológica de insectos acuáticos en las tres quebradas muestreadas fue moderada (Fig. 9, Tabla 2). Esto pudo deberse a las condiciones morfométricas, fisicoquímicas de las quebradas que no favorecen completamente a la diversidad de las especies. De acuerdo a Krebs (2000), la diversidad está determinada por diversos factores, pero sin duda uno de los principales es la estabilidad ambiental. Este factor es necesario para la conservación de la diversidad de especies, pero hay otros factores que también pueden influir en la diversidad de las especies que forman parte de la comunidad, la productividad, así como también influyen la actividad predatoria y la competencia inter e intraespecífica.

Los ambientes con mayor complejidad de hábitats pueden soportar un mayor número de especies, pues ofrecen más recursos habitacionales y alimenticios; como en el caso de las quebradas estudiadas, que se consideran valores medio altos de biodiversidad. Los resultados obtenidos al calcular el índice de Dominancia (Fig.10, Tabla 3), indican que la quebrada Jutiapa presentó el mayor índice de dominancia, siendo dominante la familia Leptophlebiidae, del orden Ephemeroptera. En segundo lugar, en la quebrada Corralitos, dominó la familia Culicidae del orden Díptera y en tercer lugar, en la quebrada Limones, la familia Leptophlebiidae fue dominante.

El índice de Similitud de Jaccard, basado en el número de especies que son exclusivas de las comunidades, en este caso exclusivas de las quebradas Limones, Corralitos y Jutiapa y en el número de especies comunes para ambas comunidades. De allí que, de acuerdo a los resultados obtenidos, la similitud es mayor entre 
quebrada Limones y quebrada Jutiapa y la disimilitud entre quebrada Corralitos y Jutiapa; esta similitud y disimilitud entre las quebradas se podría deber a la heterogeneidad ambiental de las áreas muestreadas.

Al aplicar el BMWP-CR, según se aprecia en los resultados de la Tabla 3, se define a las 3 quebradas de la microcuenca Jutiapa como de aguas de calidad regular, eutrofia y contaminación moderada con muy poca diferencia en los valores calculados, pero siempre dentro del mismo rango de acuerdo a la Tabla 3. De acuerdo a los resultados del BMWP-CR, las quebradas Limones, Corralitos y la quebrada Jutiapa presentan una calidad del agua regular, eutrofia y contaminación moderada.

Se concluye que los valores encontrados de los parámetros físico-químicos, el análisis del índice de diversidad, la dominancia y la similitud entre las quebradas estudiadas, son coincidentes en señalar que la calidad del agua es regular y con contaminación moderada. Se observó que las especies sensibles y moderadamente sensibles que pertenecen al orden Plecóptera (estuvo presente solo en quebrada Limones), Ephemeroptera y Trichoptera fueron significativamente menores en las otras quebradas, proliferando aquellas familias de gran tolerancia a la contaminación como los organismos de las familias Culicidae, Simulidae, Ceratopogonidae, Chaoboridae, Chironomidae, y muchos oligochaetos e Hirudíneos (ND) que no fueron considerados en esta investigación por tratarse de un estudio de insectos acuáticos.

Murgel, (1984) menciona que las familias anteriores son capaces de tolerar tensiones muy bajas de oxígeno por extensos períodos de tiempo, lo que particularmente resulta en una rápida tasa reproductiva (Welch, 1992). A medida que el río drena a través de los asentamientos humanos, sus aguas son extraídas para riego y consumo, lo cual se evidencia principalmente en la disminución de la velocidad de la corriente y descarga aguas abajo, siendo a su vez impactadas por la contaminación derivada de los fertilizantes. La presencia de basura no biodegradable, desechos domésticos en los alrededores y dentro del agua fue muy evidente en quebrada Corralitos; ya que es un lugar visitado por turistas.

En conclusión, las tres quebradas estudiadas son diferentes en parámetros físicoquímicos, abundancia, y levemente diferentes en composición, dominancia, diversidad de la entomofauna, y la similitud es mayor entre quebrada Limones y quebrada Jutiapa. La quebrada Limones (A) parece estar habitada por una fauna particular, probablemente adaptada a condiciones ambientales y físico-químicas poco afectadas por acciones antropogénicas, por lo que de acuerdo a los 
resultados del índice de diversidad, esta quebrada podría ser considerada como un sitio de referencia con buenas condiciones en su calidad biológica para futuras evaluaciones.

\section{RECOMENDACIONES}

En consideración a los resultados de este estudio realizado por los estudiantes de la cátedra de Entomología Acuática de la Universidad Nacional Autónoma de Honduras y a las observaciones de campo y consultas hechas a los pobladores, nos permitimos hacer las siguientes recomendaciones:

- Orientar a personas (funcionarios gubernamentales, educadores y personas interesadas) acerca de un sistema de monitoreo sencillo y eficaz para evaluar a lo largo del tiempo y en sitios permanentes, el estado y manejo apropiado de sus recursos naturales, la salud de su ambiente y el seguimiento de los cambios antropogénicos.

- Evitar los asentamientos humanos en la orilla de las microcuencas y en las zonas de recarga, para evitar el deterioro de las fuentes de agua, las cuales a su vez son utilizadas para el consumo humano.

- Elaborar un plan de trabajo para las diferentes microcuencas, que establezca el uso adecuado y el mantenimiento sostenible, para asegurar la disponibilidad de este vital liquido en los años posteriores.

- Hacer conciencia a la población adyacente a las microcuencas sobre el manejo de los residuos domésticos mediante campañas informativas, o por medio de charlas impartidas periódicamente por la alcaldía municipal para prevenir el aumento de la contaminación de las fuentes de agua.

- Educar a la población sobre la importancia de los cuerpos de agua y cómo evitar el deterioro de los mismos.

- Complementar el estudio biológico de los cursos de agua con la información físico-química ya que el estudio de las especies de fauna que están en los cursos de agua dulce se complementan con la información físico-química de las aguas, especialmente medidas de velocidad de la corriente, anchura y profundidad, tipo de sustrato, y descarga, estabilidad del lecho del río, el gradiente, área de vegetación, temperatura ambiental y del agua, turbidez, conductividad, $\mathrm{pH}$, 
Oxígeno disuelto, $\mathrm{DBO}_{5}, \mathrm{BDQ}$, dureza total, nutrientes derivados del nitrógeno (nitritos, nitratos, amonio, nitrógeno orgánico) cloro residual, fósforo total, ortofosfatos, niveles de sílice, número de bacterias coliformes y Escherichia coli presentes son también datos muy importantes. En el caso de que se sospecha contaminación por metales, se deberá evaluar la presencia de aluminio, bromo, cloro, cobre, cianuro, flúor, yodo, hierro, manganeso, molibdeno, níquel, plata y zinc que en cantidades excesivas pueden ser perjudiciales para la salud humana.

- Realizar estudios de las variaciones estacionales de la abundancia y la diversidad de insectos acuáticos y relacionarlos con la calidad del agua (BMWP$\mathrm{CR}$ ), para establecer programas de manejo ambiental adecuado, especialmente en la quebrada Corralitos.

- Hacer estudios posteriores relacionados con el tema, principalmente en la estación Limones (A), ya que está ubicada en la parte alta de la microcuenca y los parámetros de calidad son muy buenos, y puede servir como quebrada piloto.

- Establecer un programa de sanciones para todos los individuos o instituciones que contravengan las disposiciones de protección ambiental en el PNLT, sobre todo para aquellas personas que utilizan químicos y fertilizantes en los diferentes cultivos.

\section{REFERENCIAS}

Aguilar, H. Meza, N y Álvarez, G. 2004. Insectos Acuáticos Asociados al Río La Fortuna Quebradas Aledañas. Clase Entom. Acuática. Depto. Biología. UNAH. Teg. Honduras. 23 pp.

Alba-Tercedor J. 1996. Macroinvertebrados acuáticos y calidad de las Aguas de los ríos. IV Simposio del agua en Andalucía, Almería. Vol. II: 203-213.

Alba-Tercedor, J. \& A. Sánchez-Ortega. 1988. Un método rápido y simple para evaluar la calidad biológica de las aguas corrientes basado en el de Hellawell (1978). Limnética. Vol. 4:51-56.

Argueta, I. et al. 2008. Diversidad de Insectos Acuáticos en las Confluencias de la Microcuenca del Río San Juan: Quebrada Las Trojas, Quebrada Las Cañas y Quebrada Dulce. Clase Entom. Acuática. Escuela Biología. UNAH. Teg. Honduras.

Baev, P.V. \& L.D. Penev. 1995, Biodiversidad: program for calculating biological diversity parameters, similarity, niche overlap, and cluster analysis, Versión 5.1. 
Pensoft. Sofía-Moscow- 57 pp.

Borjas, G. et. Al. 1987. Tesis: Parámetros Fisicoquímicos Asociados a Macroinvertebrados Bentónicos en el río Choluteca y sus Cabeceras. Universidad Nacional Autónoma de Honduras. Tegucigalpa, Honduras. 149 pp.

Borjas, G. 1984. Monografía: Macroinvertebrados como Indicadores Biológicos de Contaminación en el río Chiquito. Tegucigalpa, Honduras. Universidad Nacional Autónoma de Honduras. Tegucigalpa, Honduras. 57 pp.

Borjas, G, et al 1997. Calidad de Agua en 6 subcuencas del Parque Nacional Azul Meámbar. Universidad Nacional Autónoma de Honduras. Tegucigalpa. Honduras. 49 pp.

Cairns, J. \& R. Pratt. 1993. A history of biological monitoring using benthic macroinvertebrates. Pages 10-27. In D. M. Rosenberg \& V. H. Resh (Eds.) Freshwater Biomonitoring and Benthic Macro-invertebrates. Chapman \& Hall, New York.

Castillo, J. 2002. Monografía: Estudio Preliminar de la Distribución de Macroinvertebrados Bentónicos y su Utilización como Indicadores de Contaminación Orgánica en la Cuenca del Río Sabacuante. UNAH-SANAA. Tegucigalpa, Honduras, 70 pp.

Chessman, B.C. 1995. Rapid assessment of river using macroinvertebrates: a Procedure based on habitat-specific sampling, family level identification and biotic index. Australian Journal of Ecology 20; 122-129

Chila. M. A. 1998. An attempt to application of benthic macro-invertebrates for the assessment of water quality. Acta Hydrobiol., Vol. 40:55-65.

Daniel, O. 2002. Página Web: iufro.boku.ac.at/iufro/iufronet/d6/

Ecología y Servicios S.A. de C.V. 1998. Caracterización, evaluación y levantamiento cartográfico de los Recursos Naturales del Parque Nacional La Tigra. Tegucigalpa. $97 \mathrm{p}$.

Forbes S. A. 1887. The Lake as a Microcosm. Bull. of the Scientific Association (Peoria, IL): 77-87.

Forbes, S.A. 1928. The biological survey of a river system-its objects, methods, and results Bulletin of Illinois Natural History Surveys. Vol. 17. No. 7: 277-284.

Forbes, S.A. and R.E. Richardson. 1913. Studies on the biology of the upper Illinois River. Bulletin of Illinois Natural History Surveys. Vol. 9., N0 10:481-574.

Gamero A. y Talavera L. 2003. Diversidad de Insectos Acuáticos en la Quebrada Las Trojas. Clase Entom. Acuática. UNAH. Teg. Honduras.

Gonzales del Tanago del Río, Marta y García de Jalón Lastra, Diego. 1995. Restauración de Ríos y Riberas. Universidad Politécnica de Madrid. 319 pp.

Hawkes, H. A. 1979. Invertebrates as indicators of river quality. In, A. James \& L. Evison, Biological indicators of water quality. John Wiley \& Sons. 2: 1-45.

Hellawell, J.M. 1986. Biological indicators of freshwater pollution and environmental management. El sevier Applied Science. London. 122p. 
Hermanson, T. 1999. An evaluation of the Peinador and biological monitoring working party macro-invertebrate indices in assessment of biological water quality in tropical streams of Golfito, Costa Rica. Adv. M. Springer. Macalester: 18pp.

Herrera, M. 2005. Guía para Evaluaciones Ecológicas Rápidas con Indicadores Biológicos en Ríos de Tamaño Mediano. Talamanca Costa Rica. Centro Agronómico Tropical de Investigación y Enseñanza (CATIE). Turrialba, Costa Rica. $866 \mathrm{pp}$

http://www.catie.ac.cr/CatieSE4/BancoMedios/Documentos\%20PDF/guía evaluaciones-pdf-Consultado: 3 de Enero del 2010

Hilsenhoff, W.L. 1977. Use of arthropods to evaluate water quality of streams. Tech. Bull. WI. Dept. Nat. Resour. No 100 15pp.

Hoja Cartográfica de Tegucigalpa Hoja 2758 II Serie 752.

INBio-TNC. 2008. Programa de Monitoreo de los Objetos de Conservación. Costa

Rica. URL: http://www.inbio.ac.cr/osa/paginas/programa.html. Consultado: 17 de Abril 2009

Junqueira, M. V., M. C. Amarante, C. F. S. Dia. \& E. S. França. 2000. Biomonitoramento da qualidades das águas da bacia do Alto Rio das velhas (MG/Brasil a través de macroinvertebrados. Acta Limnologica Brasiliensia. Vol. 12(1): 73-87.

Kolkwitz, R. \& M. Marsson. 1909. Okologie der tierischen Saprobien. Beitrage zur Lehre von der biologischen Gewasserbeurteilung. Internacionale der gesamten Hydrobiologie und Hydrographie 2:126-52. In: Hauer F. R. and G.A.

Krebs, Ch., 2000. Ecología Estudio de la Diversidad y Abundancia. Oxford. 753pp. Margalef, R. 1968. Perspectivas in ecological theory. University of Chicago Press. Marroquin, Tejeda V. B.R. Padilla y C. Medrano. 2000. Determinación de la calidad del agua de la Quebrada La Salada de La Montañita. Clase Ento. Acuatica. Depto. De Biología. UNAH. Teg. Honduras. 24 pp.

Medianero E., y M Samaniego. 2004. Comunidad de Insectos acuáticos Asociados a condiciones de contaminación en el río Curundu Panamá. Folia Entomol. Mex.,49(3):279-294 (2004).

Merritt R. W. y K.W. Cummins. 1996. An introduction to the aquatics insects of North America. 3rd Edition. Kendal/Hunt publishing Company. USA.

Mérida J. 1996. Estudio de la Calidad del Agua de la Quebrada La Salada, La Montañita. F.M. Clase Ento. Acuática. Depto. Biología. Teg. Honduras. 22 pp.

Miserendino, M. \& L. Pizzolón. 1999. Rapid assessment of river water quality using macro-invertebrates: a family level biotic index for the Patagonic Andean zone. Acta Limnologica Brasiliensia. Vol. 11, N0 2,: 137-148.

Tatumbla. F.M. Clase Ento. Acuática. Depto. Biología. UNAH. Teg. Honduras. 32 pp. Murgel, S. 1984. Limnología Sanitaria. Estudio de la polución de aguas 
continentales. Secretaría General de la Organización de los Estados Americanos. Washington, D.C. 120 pp.

Naranjo, C., G. Garcés, D. González, S. Muñoz \& Y. Musle. 2003. Una metodología rápida y de fácil aplicación para la evaluación de la calidad del agua utilizando el índice BMWP-Cu para ríos cubanos. Evento de la Facultad de Ciencias Naturales, http://www.cnt.uo.edu.cu ISBN 959-207-093-8

Consultado: 20 Abril 2010

Pyne A.I. 1986. The ecology of tropical lakes and rivers. John Wiley \& Sons, Chichester, Great Britain. $245 p$.

Richardson, R.E. 1928. The bottom fauna of the middle Illinois River, 1913-1925: its distribution, abundance, valuation, and index value in the study of stream pollution. Bulletin of Illinois Natural History Surveys. Vol. 17. No 12: 387-475.

Roldán, P. G. 2002. Bioindicación de la calidad del agua en Colombia. Uso del Método BMWP/Col. Editorial Universidad de Antioquia: 182 pp.

Roldán, G. 1999 Los macroinvertebrados y su valor como indicadores de la calidad del agua. Rev. Acad. Colomb.Cienc.23(88):375-387ISSN03703908.URL http://www.accefyn.org.co/Publi/Acad/Periodicas/Volumen3/88/375387.pdf. Consultado: 10 de Enero de 2010.

Rosenberg, D.M., H. V. Danks \& D, M. Lehmkuhl. 1986. Importance of insects in environmental impact assessment. Environmental Management. 10:773-783.

Shannon, C. \& W. Weaver. 1949. The Mathematical Theory of Communication. pp. 19-27, 82-103, 104-107. The University of Illinois Press.

Southwood, T.R.E. 1978. Ecological Methods. Second Edition. A.Hasted and Hall. John Willey \& Sons. N.Y. 524 pp.

Suess, M. J. Ed. 1982. Examination of water for pollution control. A reference handbook. Pergamon Press.3.

Welch, E.B. 1992. Ecological effects of wastewater. 2 3/4.ed. Chapman \& Hall. 425 pp.

\section{ANEXOS}

\section{Anexo\# 1}

CARACTERÍSTICAS MORFOMÉTRICAS DE LA ZONA DE ESTUDIO

\begin{tabular}{lc} 
ÁREA (Conteo de cuadros) & $45.30 \mathrm{Km}$ \\
LONGITUD DE CONTORNO & $36.89 \mathrm{Km}$ \\
LONGITUD DEL CAUCE PRINCIPAL & $5.83 \mathrm{Km}$ \\
? LONGITUD DE TRIBUTARIOS & $24.97 \mathrm{Km}$ \\
ALTURA Máx. & $2140 \mathrm{msnm}$ \\
ALTURA Mín. & $900 \mathrm{msnm}$ \\
DISTANCIA DE ALTURAS EN LINNEA RECTA & $10.212 \mathrm{Km}$ \\
ORDEN DEL RÍO (STRAHLER) & 3 \\
\hline
\end{tabular}




\begin{tabular}{l|c} 
Densidad de drenaje & $0.55 \mathrm{Km}^{-}$ \\
Constante de mantenimiento & 1.81 \\
Coeficiente de gravelius & 1.54 \\
Forma de cuenca & Oblonga \\
Pendiente de la cuenca & $0.01 \%$ \\
Pendiente del cauce prinicipal & $21.2 \%$ \\
Tiempo de concentración & $0.20 \mathrm{hrs}$. \\
Alejamiento medio & 0.86 \\
İndice de alivio & 0.12 \\
Fecuencia & $0.28 \mathrm{Km}^{2-}$ \\
\hline
\end{tabular}

\section{Anexo\#2}

\section{RANGOS DE NIVELES DE CALIDAD DE AGUA, DE ACUERDO al BMWP-CR.}

\begin{tabular}{l|c|}
\multicolumn{1}{c|}{ Nivel de calidad de agua } & BMWP-CR \\
\hline $\begin{array}{l}\text { Aguas de calidad excelentes } \\
\text { Aguas de calidad buena, no cantaminadas o no alteradas }\end{array}$ & $>120$ \\
de manera sensible & $101-120$ \\
$\begin{array}{l}\text { Aguas de calidad regular, eutrofia, contaminación } \\
\text { moderada }\end{array}$ & $61-100$ \\
\hline $\begin{array}{l}\text { Aguas de calidad mala, contaminadas } \\
\text { Aguas de calidad mala, muy contaminadas } \\
\text { Aguas de calidad muy mala, extremadamente } \\
\text { contaminadas }\end{array}$ & $36-60$ \\
\hline
\end{tabular}

\section{Anexo\#3}

\section{Materiales y Equipo de laboratorio y de campo utilizados en el estudio}

\section{Materiales y equipo de campo}

- Hoja cartográfica de Tegucigalpa

- Cámara fotográfica

- Conductímetro marca Lamotte

- Oxinómetro YSI modelo 57

- Cintas reactivas para $\mathrm{pH}$

- Cinta métrica

- Alcohol 70\%

- Tamiz y cedazos

- Pinceles

- Frascos con alcohol (colecta)

- Pinzas

- Agua destilada

\section{Materiales y equipo de laboratorio}

- Claves taxonómicas (Merrit y Cummins, 1989)

- Estereoscopios Leica 10.5 - 45X

- Cápsulas de Petri

- Agujas entomológicas para disección

- Pinceles

- Frascos plásticos (para colección permanente)

- Frascos de vidrio (preservación)

- Papel calca

- Chinógrafo 